\title{
ТИРЕОТРОПИНОМА У ПАЦИЕНТА ДЕТСКОГО ВОЗРАСТА: КЛИНИЧЕСКИЙ СЛУЧАЙ
}

\section{Коваленко Т.В., Калинченко Н.Ю., Муллахметова Л.М., Алешкевич Е.В.}

ФГБОУ ВО «Ижевская государственная медииинская академия» Минздрава России, Ижевск, Российская Федерация, ФГБУ «Национальный медицинский исследовательский иентр эндокринологии» МЗ РФ, Москва, Российская Федераџия

Причиной синдрома тиреотоксикоза в детской эндокринологии в подавляющем большинстве случаев, несомненно, является болезнь Грейвса. Однако следует принимать во внимание и возможность развития тиреотоксикоза центрального генеза, что позволит избежать серьезных лечебных ошибок. Приводим клинический случай.

Пациент Ч. впервые консультирован детским эндокринологом в возрасте 9,8 лет по поводу сердцебиения, повышения АД, эмоциональной лабильности, похудания, повышенной потливости. Болен около 4 месяцев.

Анамнез жизни: ребенок от III осложненной беременности (угроза прерывания, анемия 1 степени), роды 2 при сроке гестации 39 недель, масса тела при рождении 3500 г, длина 52 см. Ранний анамнез без особенностей. Хронической соматической патологии нет. Наследственность не отягощена, патологии эндокринной системы среди родственников I степени родства нет.

В физикальном статусе при первичном обследовании состояние средней степени тяжести, рост 153 см (+2,8 SD), масса тела 35,8 кг, ИМТ 15,3 кг/м² (-0,6 SD), общий тремор, тахикардия (до 170 ударов/минуту), повышение АД (до 130/80 мм рт. ст.), умеренный экзофтальм. Щитовидная железа увеличена (2 степень по классификации ВО3), плотная. Стадия полового развития по Таннер: P1G1, тестикулы допубертатных размеров.

Гормональные показатели: ТТГ = 25,3 мМЕ/л, СТ4 = 94,0 пмоль/л, анти-ТПО = 3,7 МЕ/л.

УЗИ щитовидной железы: расположена типично, тиреоидный объем увеличен $(22,9$ мл), структура неоднородная, эхогенность снижена, кровоток усилен.

После получения результатов обследования проводилась дифференциальная диагностика в направлении исключения редких вариантов тиреотоксикоза: тиреотропиномы, резистентности к тиреоидным гормонам. В связи с этим проведено МРТ головного мозга, диагностирована микроаденома гипофиза.

Гиперсекреции других гормонов гипофиза не выявлено: ЛГ = 1,44 мМЕ/мл, ФСГ = 4,74 мМЕ/мл, пролактин $=111,2 \mathrm{ME} /$ л.

С предположением на тиреотропиному направлен в НМИЦ эндокринологии МЗ РФ, первичная госпитализация состоялась в июне 2018 года (в возрасте 9,9 лет). Диагноз тиреотропиномы аргументирован по результатам клинико-лабораторного и инструментального обследования:

- наличие клинических симптомов тиреотоксикоза;

- высокий уровень СТ4, СТ3 (до 7,7 пмоль/л), ТТГ (до 45,1 мМЕ/л);

- микроаденома гипофиза по данным МРТ головного мозга (в центральной части аденогипофиза определяется образование овальной формы, размерами $5 * 4,5 * 4$ мм, умеренно гипоинтенсивное на Т1взвешенных изображениях, при контрастном усилении характеризующееся сниженным (по сравнению с тканью аденогипофиза) накоплением контрастного препарата). 
Проведено молекулярно-генетическое обследование, выявлено отсутствие мутации в гене рецептора к тиреоидным гормонам (THRß). Рекомендовано оперативное лечение.

До проведения операции получал медикаментозную терапию: Тирозол в дозе 30-10 мг/сут, Анаприлин, препарат сандостатина (Октреотид 100 мг 3 раза в сутки, затем Октреотид-лонг 20 мг в/м).

В возрасте 10,2 лет в НМИЦ эндокринологии МЗ РФ проведена операция: трансназальное трансфеноидальное удаление эндоселлярной аденомы гипофиза (тиреотропиномы). По поводу послеоперационного транзиторного гипотиреоза получал левотироксин 50 мкг/сут в течение 1 года.

Пациент продолжает наблюдение у детского эндокринолога. По результатам консультации в возрасте 13,2 лет: состояние удовлетворительное, рост 170 см (+1,89 SD), масса тела 55 кг, ИМТ 19,03

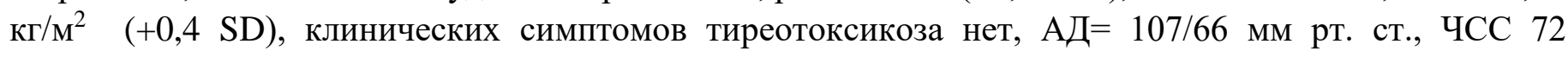
удара/минуту. Щитовидная железа увеличена (2 степень по классификации ВО3). Стадия полового развития по Таннер: P4G4, тестикулы 15 мл.

Тиреоидный профиль: ТТГ = 0,79 мМЕ/л, СТ4 = 14,3 пмоль/л. Регулярный контроль МРТ головного мозга с внутривенным контрастированием 1 раз в 6-12 месяцев не выявил продолженного роста опухоли.

Представленный клинический случай демонстрирует редкий для педиатрической практики вариант тиреотоксикоза - ТТГ-секретирующую аденому гипофиза. Диагностический поиск в этом направлении был обусловлен выявлением повышенного уровня ТТГ у пациента с тиреотоксикозом. Определенную сложность при этом вызывали два момента. Первый из них - наличие диффузного зоба у обследованного, что могло ошибочно трактоваться как проявление болезни Грейвса. Однако литературные данные свидетельствуют о высокой частоте (до 80-90\%) увеличения щитовидной железы при тиреотропиномах. Второй момент, который учитывался при проведении дифференциальной диагностики - это наличие у пациента микроаденомы гипофиза, тогда как тиреотропиномы чаще ассоциированы с макроаденомами гипофиза. Это потребовало проведения молекулярно-генетического исследования для исключения синдрома резистентности к тиреоидным гормонам, при котором микроаденома могла быть случайной находкой и трактоваться как инцидентолома.

В лечении тиреотропином методом выбора является оперативное лечение, в результате которого у обсуждаемого пациента достигнута ремиссия заболевания. 